\title{
Experimental infection and co-infection of dogs with Anaplasma platys and Ehrlichia canis: hematologic, serologic and molecular findings
}

\author{
SD Gaunt ${ }^{1}$, MJ Beall ${ }^{*}$, BA Stillman², L Lorentzen ${ }^{2}$, PPVP Diniz ${ }^{3}$, R Chandrashekar ${ }^{2}$, EB Breitschwerdt ${ }^{4}$
}

\begin{abstract}
Background: Rhipicephalus sanguineus is a ubiquitous tick responsible for transmitting Ehrlichia canis and most likely Anaplasma platys to dogs, as either single or co-infections. The objective of this study was to assess the effects of either simultaneous or sequential experimental infections with E. canis and A. platys on hematological and serological parameters, duration of infection, and efficacy of doxycycline therapy in dogs infected with one or both organisms. Six dogs per group were either uninfected, A. platys infected, E. canis infected, A. platys and E. canis co-infected, A. platys infected and E. canis challenged or E. canis infected and A. platys challenged at day 112 post-infection (PI). Doxycycline treatment was initiated at 211 days $\mathrm{Pl}$, followed by dexamethasone immunosuppression beginning 410 days PI.

Results: Initially, transient decreases in hematocrit occurred in all groups infected with E. canis, but the mean hematocrit was significantly lower in the A. platys and E. canis co-infected group. All dogs except the controls developed marked thrombocytopenia after initial infection followed by gradually increased platelet counts by 112 days PI in groups with the single infections, while platelet counts remained significantly lower in the A. platys and E. canis co-infected group. Both sequential and simultaneous infections of $A$. platys and $E$. canis produced an enhanced humoral immune response to A. platys when compared to infection with A. platys alone. Likewise, coinfection with $E$. canis and $A$. platys resulted in a more persistent $A$. platys infection compared to dogs infected with A. platys only, but nearly all A. platys infected dogs became A. platys PCR negative prior to doxycycline treatment. E. canis infected dogs, whether single or co-infected, remained thrombocytopenic and E. canis PCR positive in blood for 420 days. When treated with doxycycline, all E. canis infected dogs became E. canis PCR negative and the thrombocytopenia resolved. Despite immunosuppression, neither A. platys nor E. canis DNA was PCR amplified from doxycycline-treated dogs.

Conclusions: The results of this study demonstrate that simultaneous or sequential infection with A. platys and E. canis can alter various pathophysiological parameters in experimentally infected dogs, and because natural exposure to multiple tick-borne pathogens occurs frequently in dogs, awareness of co-infection is important in clinical practice.
\end{abstract}

\section{Background}

Ehrlichia canis is a Gram-negative, obligate intracellular bacterium which infects monocytes and is the primary causative agent of canine monocytic ehrlichiosis [1]. Rhipicephalus sanguineus transmits E. canis to dogs both transtadially and intrastadially [2]. Canine infections caused by E. canis are more commonly reported in the

* Correspondence: melissa-beall@idexx.com
${ }^{2}$ IDEXX Laboratories, Inc. Westbrook, ME, USA southern regions of the United States, however R. sanguineus is distributed throughout the country [3]. Experimentally, infection with $E$. canis results in acute, subclinical and chronic disease stages with dogs having a variety of clinical signs and laboratory abnormalities including fever, lethargy, lameness, oculonasal discharge, thrombocytopenia, non-regenerative anemia, leukopenia, hyperglobulinemia and proteinuria during various stages of infection. Often, chronic infection with E. canis will go unrecognized because infected dogs appear healthy 
until late in the infection when pancytopenia, uveitis, weight loss and hemorrhagic disorders arise, and a diagnosis of ehrlichiosis is made [1].

Canine cyclic thrombocytopenia is caused by Anaplasma platys, a Gram-negative, obligate intracellular bacterium that infects platelets [1]. The dog is the primary reservoir host for A. platys and to date, this organism has not been shown to infect humans. A. platys is likely transmitted by the $R$. sanguineus tick, however experimental infection studies have not conclusively demonstrated transmission [4]. A. platys infections are often found in the same geographic regions as $E$. canis and evidence of exposure to or infection with both organisms is often detected in the same dog [5-8]. Both organisms are found on all continents throughout the world, but are more prevalent in tropical and subtropical climates [2,9]. Case reports and case series, incorporating PCR-based modalities have confirmed co-infections with $E$. canis and A. platys $[8,10]$. Experimentally, $A$. platys infections cause a cyclic thrombocytopenia that may be severe enough to result in bleeding, including petechiae and ecchymoses, but most dogs are thought to control the infection immunologically [1].

Given that $E$. canis and A. platys likely share the same tick vector, $R$. sanguineus, dogs may become infected with both organisms, either simultaneously or sequentially. Most dogs infested with $R$. sanguineus have numerous attached ticks. The clinical impact of an $E$. canis and A. platys co-infection on the pathophysiology of disease in dogs has not been thoroughly investigated. A previous study has shown that naturally infected clinically ill dogs, suspected of having either Lyme disease, granulocytic anaplasmosis, or both diseases, were nearly twice as likely to have antibodies to both Borrelia burgdorferi and A. phagocytophilum as compared to healthy dogs from the same region, suggesting that exposure to more than one pathogen may increase the likelihood of disease expression [11]. The objective of this study was to assess the effects of either simultaneous or sequential infections with $E$. canis and A. platys on hematological and serological parameters, the duration of infection, and the comparative efficacy of doxycycline therapy in the dogs infected with one or both organisms.

\section{Methods}

\section{Inoculum}

An A. platys isolate originating from blood of a dog with uveitis, thrombocytopenia and morulae in platelets was used to infect dogs in this study [12]. Several prior experimental infections of dogs with this isolate have been reported [13,14]. For the inoculum, blood from a splenectomized dog inoculated with this $A$. platys isolate 10 days before was collected into $3.8 \%$ citrate. Plateletrich plasma was prepared, $10 \%$ DMSO added, and $2 \mathrm{~mL}$ aliquots were stored in liquid nitrogen until administration. The number of platelets containing $A$. platys inclusions or morulae in this platelet-rich plasma was approximately 35\%. After storage of less than 6 months, the vials were thawed to ambient temperature and the entire $2 \mathrm{~mL}$ aliquot was administered within 1 hour into the cephalic vein of each dog.

The E. canis isolate originated from the blood of a different dog in Louisiana with fever and thrombocytopenia. Experimental canine infections, using this culture grown isolate in canine histiocytic cells, were described in two previous studies $[15,16]$. The E canis infected cells were harvested after approximately 5 days of in vitro growth when $>80 \%$ of cells contained ehrlichial inclusions as judged by a Wright-stained cytocentrifuged smear. Ten percent DMSO was added to a suspension of the cultured E. canis-infected DH82 cells, after which $2 \mathrm{~mL}$ aliquots were stored in liquid nitrogen for less than 12 months. At the time of inoculation, the vials were thawed to ambient temperature and the $2 \mathrm{~mL}$ aliquot administered through the cephalic vein of each dog within 1 hour of thawing.

\section{Dogs}

Six month old, female hound-type dogs were inoculated intravenously with $A$. platys and/or E. canis organisms. Six groups of six dogs each were evaluated: non-infected controls, A. platys infected (A), E. canis infected (E), A. platys and $E$. canis co-infected $(\mathrm{A}+\mathrm{E}), A$. platys infected followed by administration of $E$. canis 112 days later $(\mathrm{A} \rightarrow \mathrm{E})$, and $E$. canis infected followed by administration of A. platys 112 days later $(\mathrm{E} \rightarrow \mathrm{A})$. Doxycycline treatment $(10 \mathrm{mg} / \mathrm{kg}$ PO daily for 28 days) was administered to half the dogs in each group beginning at 211 days post-infection (PI). To assess treatment efficacy, all dogs were subsequently immunosuppressed by administering dexamethasone 0.3 $\mathrm{mg} / \mathrm{kg}$ IM daily for 5 days beginning at day 410 of the study. The timing of the challenge infection, administration of doxycycline, and dexamethasone immunosuppression was chosen based upon stabilization of platelet counts for infected dogs and the findings of a previous experimental infection using this isolate of E. canis [17]. The study duration was 485 days for all groups.

Whole blood and serum were collected at twice weekly, weekly or every other week intervals for 15 months after infection. Aspirates of the popliteal or prescapular lymph nodes were obtained pre- and postimmunosuppression (day 400 and 414, respectively), while bone marrow samples were obtained as aspirates collected from the iliac crest using a 16 gauge Osgood marrow needle and aseptic technique post-immunosuppression (day 414).

Physical exams that included rectal temperatures, were performed twice weekly for six weeks following each inoculation and weekly thereafter. The dogs were 
housed indoors in climate-controlled kennels at a facility accredited by the American Association for Laboratory Animal Science. The study was approved by the Institutional Animal Care and Use Committee (protocol \#0652) at Louisiana State University.

\section{Hematology}

Blood was collected into $2 \mathrm{~mL}$ vacutainer tubes containing potassium EDTA and then quickly inverted to avoid platelet clumping. Blood samples were analyzed within 3 hours of collection using a Bayer Advia 120 to measure hematocrit, mean cell volume (MCV), mean platelet volume (MPV), and platelet and total leukocyte concentrations. Prior to analysis, each blood sample was inspected for clots; any sample with visible clots was discarded and another blood sample collected. Wrightstained blood smears were also prepared from these blood samples and reviewed for platelet aggregation. Quality control procedures for the hematology instrument included daily intralab control reagents, monthly participation in the Bayer CHECKpoint Interlab QC Program, and quarterly participation in external assurance program offered by the Veterinary Laboratory Association.

\section{Serology}

All serum samples through day 420 were tested for antibodies to $E$. canis and $A$. platys using the Canine SNAP ${ }^{\circ}$ $4 \mathrm{Dx}^{\oplus}$ Test kit (IDEXX Laboratories, Inc., Westbrook, ME) according to the product insert. This multivalent ELISA (enzyme-linked immunosorbent assay) uses synthetic peptide reagents to independently detect serum antibodies to Anaplasma spp. (e.g. A. platys, A. phagocytophilum) and to E. canis. Following addition of test serum and development of the color reaction, the intensity of the color was semiquantitated by densitometry (RCP Densitometer, Tobias Associates, Ivyland, PA). The difference in optical density (OD) between the test spot color reaction and the white background on the test strip (blank) was recorded as a relative OD between 0.0 and 1.0. Although the test is not licensed for semiquantitative interpretation, a previous study has demonstrated a positive correlation between the optical density of the $E$. canis test spot and the inverse IFA titer for $E$. canis in dogs experimentally infected with E. canis [18].

\section{Polymerase Chain Reaction (PCR) Testing}

Molecular evidence of infection was assessed by two independent laboratories. The first laboratory (IDEXX Laboratories, Westbrook, ME) performed real-time PCR on whole blood collected throughout the study (Days 0$154,183,218,246,275,303,400,414,420)$, lymph node aspirates collected pre- and post-immunosuppression, and bone marrow collected post-immunosuppression.
Whole blood samples $(200 \mu \mathrm{l})$ were processed for DNA (100 $\mu$ l elution volume) using an automated system (MagNA Pure, Roche) while DNA from bone marrow and lymph node aspirates was extracted manually using a commercially available kit (HighPure Kit, Roche) according to the product insert.

A real-time PCR, hybridization probe assay was developed to detect an A. platys $p 44$ polynucleotide [GenBank:GP282016] from genomic DNA [19]. Real-time PCR was performed using a LightCycler 480 genotyping master mix (Roche) in a 20 ul volume reaction with $5 \mathrm{ul}$ of template DNA. Primers (Table 1) were used at a concentration of $0.3 \mu \mathrm{M}$ for the forward primer and $0.6 \mu \mathrm{M}$ for the reverse primer. Both probes were used at a concentration of $0.3 \mu \mathrm{M}$. PCR was performed under the following conditions: a single hot-start cycle at $95^{\circ} \mathrm{C}$ for 10 minutes followed by 50 cycles of denaturation at $95^{\circ} \mathrm{C}$ for 30 seconds, annealing at $58^{\circ} \mathrm{C}$ for 20 seconds, and extension at $72^{\circ} \mathrm{C}$ for 10 seconds. A melting curve was performed by heating the PCR product to $95^{\circ} \mathrm{C}$ for 1 minute, cooling to $45^{\circ} \mathrm{C}$ for one minute, and then gradually heating to $80^{\circ} \mathrm{C}$. Positive samples were identified from the software as having both positive crossing points and a melting curve temperature of $66.5^{\circ} \mathrm{C}$ $+/-1^{\circ} \mathrm{C}$. Analytical sensitivity was determined to be at least 10 gene copies per reaction in negative canine genomic DNA based on serial dilutions of the control plasmid. The $A$. platys $p 44$ PCR detected strains of A. platys from across the US, the Caribbean and Brazil. The A. platys p44 PCR did not detect A. phagocytophilum $p 44$ DNA from a control plasmid containing the A. phagocytophilum 44 template or A. phagocytophilum PCR-positive field samples.

An Ehrlichia spp. real-time PCR targeting the groEL gene of three Ehrlichia species [GenBank: U96731 (E. canis), AF195273 (E. ewingii), L10917 (E. chaffeensis)] was developed based on published primers [20], however using a hydrolysis probe format. Real-time PCR was performed using Lightcycler 480 probes master mix

Table 1 Primers and probes used for the A. platys (Apl) and Ehrlichia spp. (Ehr) PCR assays [19,20].

\begin{tabular}{ll}
\hline Name & Sequence $\left(\mathbf{5}^{\prime}\right.$ to $\left.\mathbf{3}^{\prime}\right)$ \\
\hline Apl forward primer & CCGGCGTTTAGTAAGATAAATG \\
\hline Apl reverse primer & GCAAATTAACGATCTCCGCC \\
\hline Apl probe 1 FITC & ACAGTATCGGGGTAGCGAGAGTAGAA \\
\hline Ehr1 forward primer & GGAGATCGGCTATGAACAGTTCAAGAC \\
\hline Ehr2 reverse primer & TCGCAGTTAAAATAGAACATGTAGTTG \\
\hline Ehr3 forward primer & CAGAGTGCTTCTCAATGTAACGA \\
\hline Ehr4 reverse primer & TTGCGGTTAAGATAGAACATGTAGTTG \\
\hline Roche UPL probe \#9 & Catalog \# 04685075001 \\
\hline
\end{tabular}


(Roche) in a $20 \mathrm{ul}$ volume reaction with $200 \mathrm{nM}$ of each of the four primers (Table 1) and UPL probe 9 (Roche) and $4 \mathrm{ul}$ of template DNA. PCR was performed under the following conditions: a single hot-start cycle at $95^{\circ} \mathrm{C}$ for 5 minutes followed by 50 cycles of denaturation at $95^{\circ} \mathrm{C}$ for 10 seconds, annealing at $60^{\circ} \mathrm{C}$ for 20 seconds, and extension at $72^{\circ} \mathrm{C}$ for 5 seconds with a single acquisition.

Conventional PCR assays, designed to detect $A$. platys, and $E$. canis infection, were performed at the second laboratory (Intracellular Pathogens Research Laboratory at North Carolina State University) on the pre- and post-immunosuppression samples. These samples included whole blood, bone marrow and lymph node aspirates. Total DNA was automatically extracted using a Qiagen robot from $200 \mu \mathrm{l}$ of blood with a commercially available kit (MagAttract DNA Blood kit, Qiagen, Valencia, CA). The final eluted volume was $200 \mu \mathrm{l}$ per sample. The DNA concentration was quantified by spectrophotometry, and absence of PCR inhibitors demonstrated by the amplification of GAPDH (glyceraldehyde3-phosphate dehydrogenase) [21]. Samples were initially screened using $16 S$ rRNA oligonucleotide primers designed to amplify all Anaplasma and Ehrlichia species [17]. The E. canis PCR assay was performed as previously described [22]. The A. platys $16 S$ rRNA and groEL genes were targeted as described previously [11]. The limit of detection, as determined by positive control plasmid dilution for each target, was: $16 S \mathrm{rRNA}=10$ gene copies per reaction and groEL gene $=5$ gene copies per reaction.

In order to prevent PCR amplicon contamination, sample extraction, reaction setup, PCR amplification and amplicon detection were performed in separated areas. Negative water controls were included with each run as was a dilution of the positive control plasmid.

\section{Statistical analysis}

The hematology data were evaluated with one-way ANOVA and Tukey's multiple comparison test to compare each group at each time point to detect significant differences $(p \leq 0.05)$. A software program (GraphPad Prism v.5, GraphPad Software, La Jolla, CA) was used to perform these analyses. A t-test was performed for serology and PCR results using statistical software (JMP8, SAS, Cary, NC). Agreement between the results of the two PCR assays was calculated by dividing the number of sample results in agreement by the total number of samples tested.

\section{Results}

\section{Hematology and clinical signs}

Compared to the non-infected controls, dogs infected with E. canis (Group E) developed decreased hematocrits, while the hematocrits of dogs infected with $A$. platys (Group A) did not differ from controls (Fig. 1a). Dogs that were co-infected with A. platys and E. canis (Group $\mathrm{A}+\mathrm{E}$ ) also developed decreased hematocrits relative to the control dogs (Fig. 1a). At several time points (days 7, 84, and 112), their hematocrits were significantly lower than dogs infected with E. canis alone (One-way ANOVA, $\mathrm{p} \leq 0.05$ ). Likewise, dogs initially infected with $A$. platys and challenged with $E$. canis at day 112 (Group $\mathrm{A} \rightarrow \mathrm{E}$ ) had a marked decrease in hematocrit following the challenge infection, whereas there was no anemia when $E$. canis infected dogs were challenged with $A$. platys at day 112 (Group E $\rightarrow$ A; Fig. 1b).

All dogs in groups $\mathrm{A}, \mathrm{E}$ and $\mathrm{A}+\mathrm{E}$ developed severe thrombocytopenia within 7 days compared to noninfected, control dogs (Fig. 2a). While the platelet counts in the Group A dogs (A. platys only) gradually increased after 75 days $\mathrm{PI}$, the platelet counts in the coinfected dogs (Group A+E) remained significantly lower

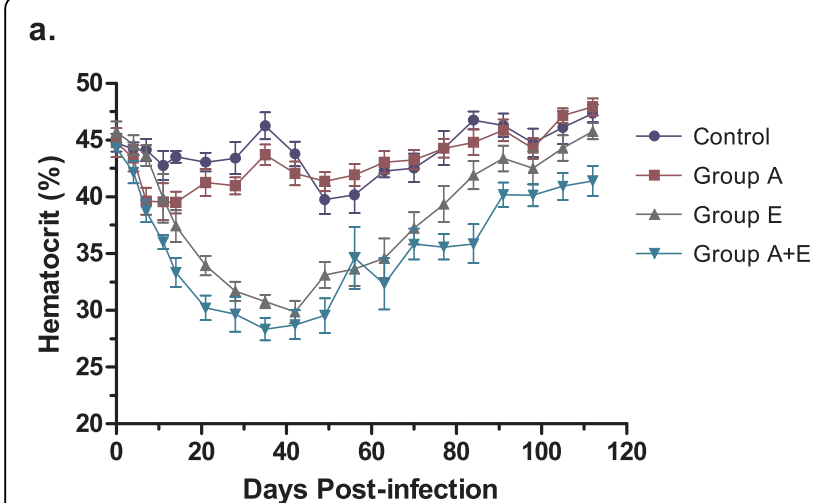

b.

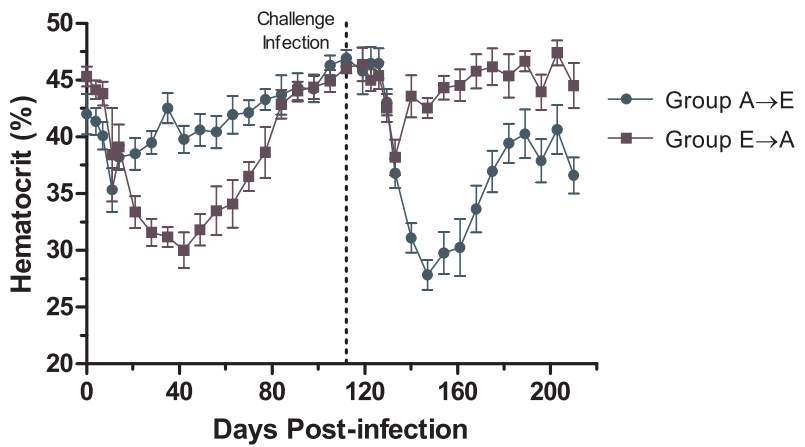

Figure 1 Effect of $A$. platys and/or E. canis infections on hematocrits of dogs prior to doxycycline treatment. (a.) Uninfected controls are compared to single infections of A. platys (Group A), E. canis (Group E) or simultaneous infections of both A. platys and E. canis (Group A+E). (b.) Groups receiving sequential infections of $A$. platys followed by $E$. canis (Group $A \rightarrow E$ ) and E. canis followed by A. platys $(G r o u p ~ E \rightarrow A)$, with the challenge infection at 112 days PI (dotted line). Controls shown in panel A. (Hematocrit shown as mean \pm SEM per group.) 

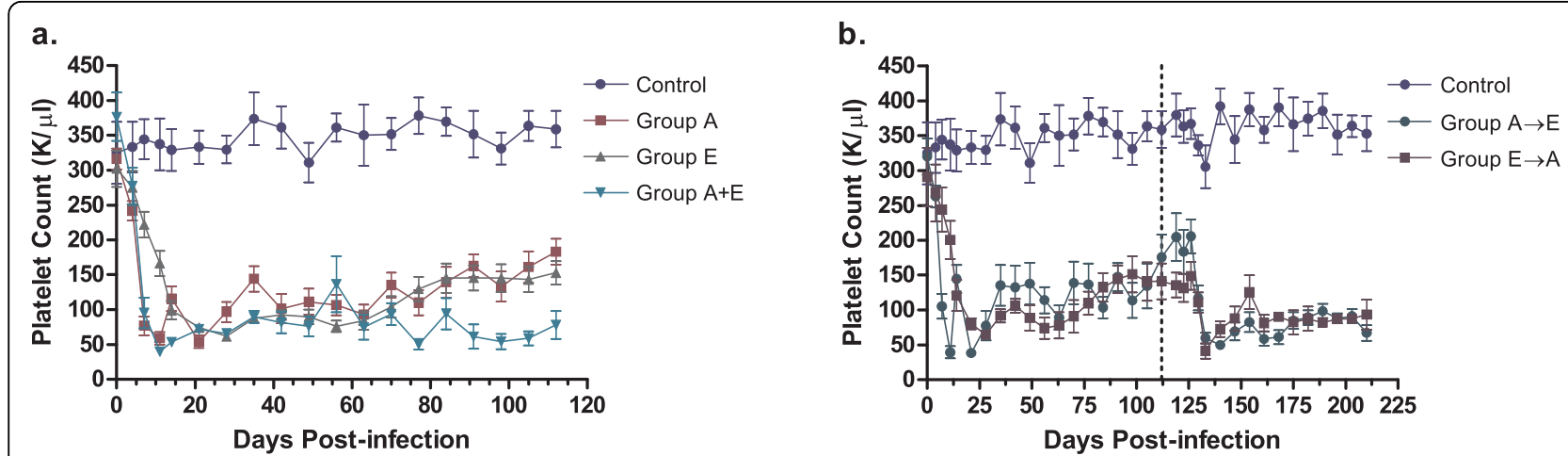

Figure 2 Effect of $\boldsymbol{A}$. platys and/or $\boldsymbol{E}$. canis infections on platelet counts of dogs prior to doxycycline treatment. (a.) Uninfected controls are compared to single infections of A. platys (Group A), E. canis (Group E) or simultaneous infections of both A. platys and E. canis (Group A+E). (b.) Uninfected controls are compared to groups receiving sequential infections of A. platys followed by $E$. canis (Group $A \rightarrow E$ ) and $E$. canis followed by A. platys (Group $\mathrm{E} \rightarrow \mathrm{A}$ ), with the challenge infection at 112 days PI (dotted line). (Platelet counts shown as mean \pm SEM per group).

than Group A platelet counts at several time points (days 77, 98-130, 144-158, 171-203; One-way ANOVA, $\mathrm{p} \leq 0.05)$. In comparison to $E$. canis infected dogs (Group E), the Group A+E platelet counts were also significantly lower at several time points (days 7, 11, 63, 77, 84, 120, 192; One-way ANOVA, $\mathrm{p} \leq 0.05$ ). In dogs that were infected sequentially with these agents (Group $\mathrm{A} \rightarrow \mathrm{E}$ and Group $\mathrm{E} \rightarrow \mathrm{A}$ ), the platelet concentrations decreased following the inoculation of the second organism (Fig. 2b). However, there was no significant difference between these two groups in the severity of the thrombocytopenia, regardless of which organism was initially and subsequently administered.

Compared to the non-infected controls, the total leukocyte counts were significantly decreased in dogs from groups $\mathrm{E}$ and $\mathrm{E}+\mathrm{A}$ between days 14-49 PI (One-way ANOVA, $\mathrm{p} \leq 0.05$ ), however leukocyte counts did not differ between these two groups (data not shown). The leukocyte counts of dogs infected with A. platys (Group A) did not differ from controls at any time point PI.

Clinically, none of the dogs developed an acute illness following inoculation with A. platys and/or E. canis organisms. Compared to controls, rectal temperatures were increased in E. canis infected dogs (Group E and $\mathrm{A}+\mathrm{E}$ ) between 21-35 days PI, but dogs infected with both A. platys and E. canis (Group $\mathrm{A}+\mathrm{E}$ ) were not significantly different from dogs infected with only $E$. canis (Group E).

\section{Real-time PCR Testing}

Within three to five days PI, A. platys DNA was amplified by PCR in all dogs inoculated with this organism. In contrast, E. canis DNA was detected between seven and fourteen days PI (mean 10 days) from the E. canis infected dogs. All E. canis infected dogs consistently tested positive by PCR between day 14 and initiation of doxycycline therapy on day 211 . In contrast, the majority of $A$. platys infected dogs became PCR negative prior to doxycycline treatment including those in Group $\mathrm{E} \rightarrow \mathrm{A}$. Co-infection with E. canis (Group A+E), however, appeared to prolong the duration of active A. platys infection. The median duration of infection for the dogs in Group A was 104 days whereas the co-infected dogs in Group $\mathrm{A}+\mathrm{E}$ had a median duration of $A$. platys infection of 119 days, excluding one dog from Group $\mathrm{A}+\mathrm{E}$ that was A. platys PCR-positive for the duration of the study.

\section{Serology}

On average, antibodies to Anaplasma spp. were first detected in the dogs inoculated with $A$. platys (Groups $\mathrm{A}$ and $\mathrm{A} \rightarrow \mathrm{E}$ ) by day 16 PI (S.D. 4.4 days; range 10-24 days). On average, groups $\mathrm{E}$ and $\mathrm{E} \rightarrow \mathrm{A}$ dogs had a detectable antibody response to the $E$. canis antigens 24 days PI (S.D. 4 days, range 17-35 days). However, coinfected dogs (Group A+E) had a delayed humoral immune response to $A$. platys antigens, with $A$. platys antibodies first detectable on average 27 days PI (S.D. 10.3 days, range 14-35 days), while the $E$. canis antibody response was similar to dogs infected with $E$. canis only (avg. 24 days PI; S.D. 7.6 days). In the two groups that received challenge infections (Group $\mathrm{A} \rightarrow \mathrm{E}$ and Group $\mathrm{E} \rightarrow \mathrm{A}$ ), the time between receiving the second inoculum and a measurable antibody response to antigens of the challenge infection averaged 28 days (range 14-35 days) regardless of whether the second inoculum consisted of A. platys or E. canis.

Serologic results from SNAP 4Dx were semiquantitated by optical densitometry for all dogs through 420 days of the study, allowing the graphical representation of the humoral immune response over time. Group A dogs, regardless of doxycycline treatment, had a steady 
decline in OD values after reaching an initial peak OD around 75 days PI with 5/6 dogs testing Anaplasma seronegative by day 420 (Fig. 3a). A. platys infected dogs that were subsequently challenged with E. canis (Group $\mathrm{A} \rightarrow \mathrm{E}$ ) had a marked increase in their OD values for $A$. platys within two weeks of receiving the $E$. canis inoculum (Fig. 3a). Like the Group A dogs, the OD values for the Group $\mathrm{A} \rightarrow \mathrm{E}$ dogs, regardless of doxycycline treatment, showed a steady decline in the Anaplasma OD through day 420 of the study with 5/6 dogs Anaplasma seronegative at that time point (Fig. 3a). The serologic response to Anaplasma antigens was influenced by coinfection (Group $\mathrm{A}+\mathrm{E}$ ) such that the A. platys OD values were significantly greater in the co-infected group as compared to dogs in Group A between 80 and 160 days PI (t-test, $\mathrm{p}<0.0001)$. Compared to the untreated co-infected dogs, $A$. platys $\mathrm{OD}$ values declined in the co-infected dogs receiving doxycycline therapy, with $2 / 3$ dogs seronegative at day 420 (Fig. 3b).

All dogs receiving the $E$. canis inoculum had a steady increase in E. canis OD values on SNAP 4Dx, reaching peak levels approximately 100 days PI. E. canis OD values remained elevated throughout the course of the study independent of doxycycline therapy (Fig. 4). Dogs in the control group remained $A$. platys and E. canis seronegative on SNAP 4Dx for the duration of the study.

\section{Doxycycline Treatment and Immunosuppression}

Doxycycline was administered to $3 / 6$ dogs in each group, including uninfected controls, for a total of 28 days beginning at day 211 of the study. In order to better assess the efficacy of doxycycline following the course of treatment, all dogs were given an

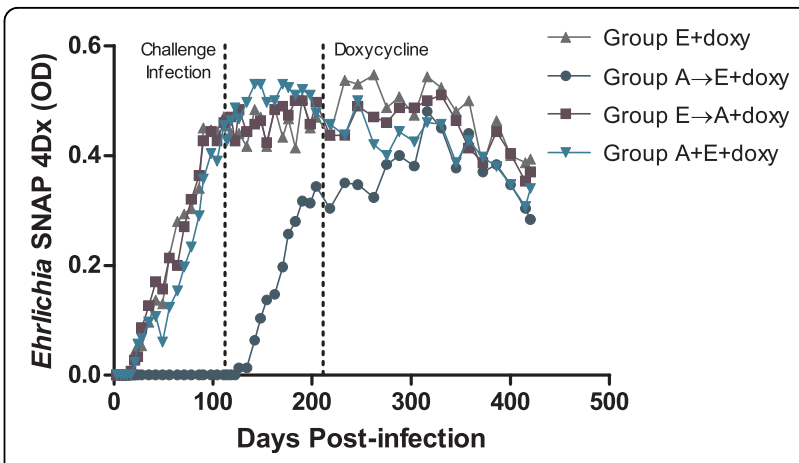

Figure 4 SNAP 4Dx OD values for $E$. canis remain elevated through day $\mathbf{4 2 0}$ of the study. Comparison of mean $O D$ values for dogs infected only with E. canis (Group E), infected with A. platys and challenged with $E$. canis or infected with $E$. canis and

challenged with $A$. platys (Groups $A \rightarrow E$ and $E \rightarrow A$ ) at day 112, and dogs co-infected with A. platys and E. canis (Group A+E). Three of six dogs in each group were treated with doxycycline beginning at day 211 (+doxy) while the other three dogs per group served as untreated controls (not shown). (Mean OD per group.)

immunosuppressive dose of dexamethasone for 5 days beginning at day 410 of the study. Physical examinations and complete blood counts were monitored through day 485. Prior to doxycycline, the uninfected controls and Group A had platelet counts within the laboratory reference interval, while all other groups $(E, A \rightarrow E, E \rightarrow A$, and $E+A$ ) were thrombocytopenic (mean $125,600 / \mu \mathrm{l}$ ). Over the next three months, average platelet counts increased in those thrombocytopenic dogs treated with doxycycline (Fig. 5a). Dogs in groups $\mathrm{E}, \mathrm{A} \rightarrow \mathrm{E}, \mathrm{E} \rightarrow \mathrm{A}$, and $\mathrm{E}+\mathrm{A}$ that did not receive doxycycline remained thrombocytopenic at day 410 (mean 130, 500/ $\mu$ l; Fig. $5 b)$. Immunosuppression with dexamethasone resulted in a transient increase in platelet counts for all dogs, but

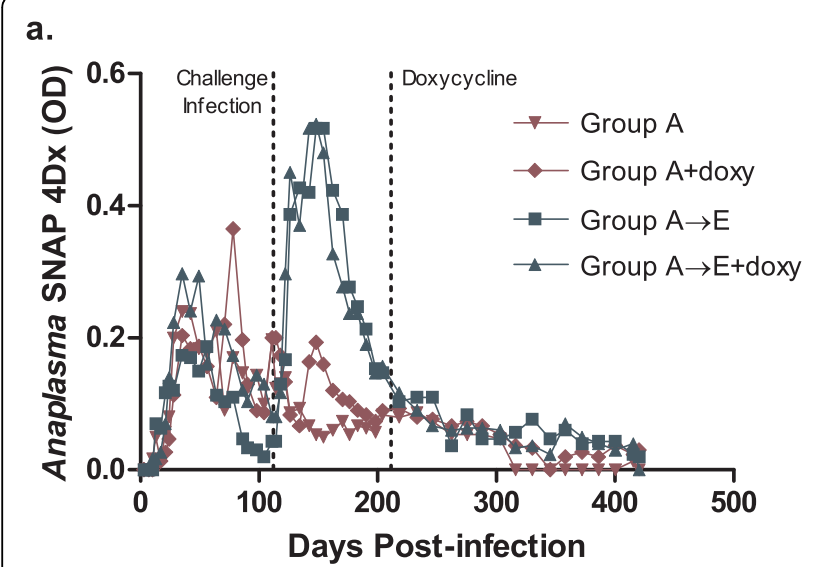

b.

Figure 3 SNAP 4Dx OD values for Anaplasma spp. vary with E. canis co-infection and doxycycline treatment. (a.) Comparison of only $A$. platys infected (Group A) to A. platys infected challenged with E. canis on day 112 PI (Group A $\rightarrow$ E). Three dogs from each group were either treated with doxycycline starting at day 211 (+doxy) or not treated. (b.) Comparison of Anaplasma spp. OD values for co-infected dogs (Group A + E). Three dogs from this group were treated with doxycycline at day $211 \mathrm{PI}$ (+doxy) and three dogs were left untreated. (Mean OD per group). 

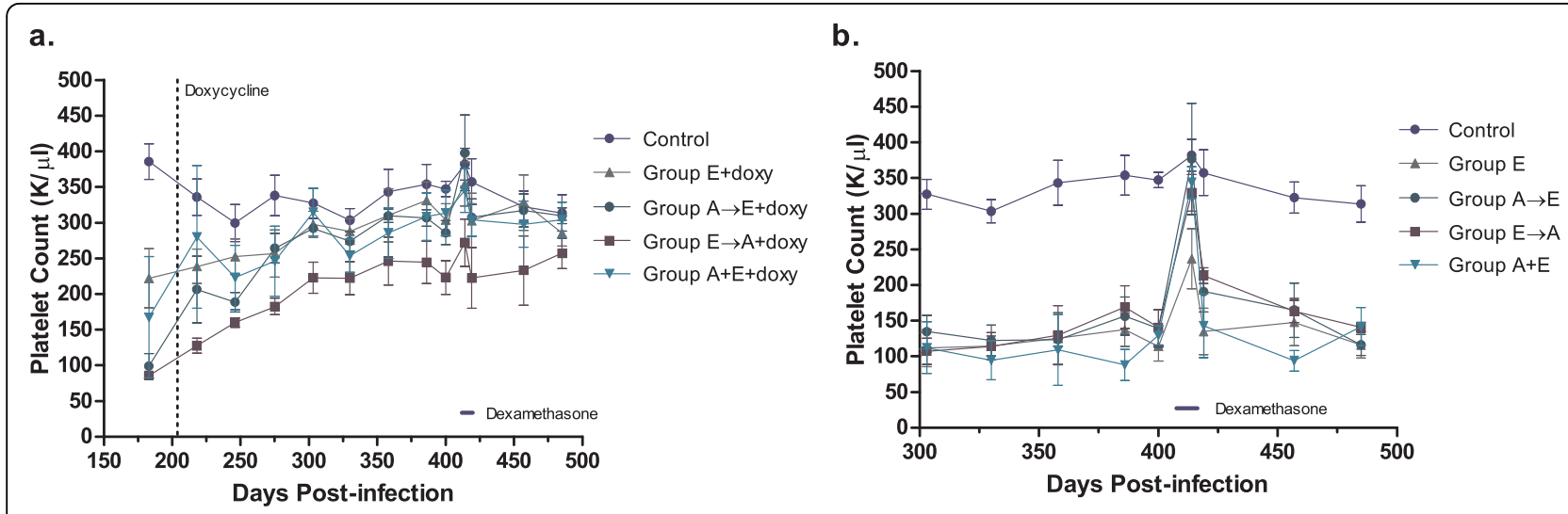

Figure 5 Differences in platelet counts in dogs infected with A. platys and/or E. canis and receiving doxycycline treatment (a.) relative to their untreated controls (b). Three dogs from each group were either treated with doxycycline starting at day 211 (+doxy) or not treated. All doxycycline treated and untreated dogs were administered dexamethasone between days 410 and 414 PI. (Platelet counts shown as mean \pm SEM per group.)

the effect was most pronounced for those dogs that were thrombocytopenic prior to immunosuppression and had not been treated with doxycycline (Fig. 5b). By day 485 of the study, the platelet counts of the dogs that did not receive doxycycline in groups $\mathrm{E}, \mathrm{A} \rightarrow \mathrm{E}$, $\mathrm{E} \rightarrow \mathrm{A}$, and $\mathrm{E}+\mathrm{A}$ were significantly lower than the platelet counts of the dogs in these respective groups that had been treated with doxycycline ( $\mathrm{t}$-test, $\mathrm{p}<0.0001$ ).

In addition to resolving the thrombocytopenia, doxycycline treatment appeared to successfully clear the dogs of any PCR evidence of the E. canis infection in blood, bone marrow and lymph node. At day 183 of the study, prior to doxycycline treatment, all dogs from groups $\mathrm{E}$, $\mathrm{A} \rightarrow \mathrm{E}, \mathrm{E} \rightarrow \mathrm{A}$ and $\mathrm{E}+\mathrm{A}$ were $\mathrm{PCR}$ positive for $E$. canis DNA. Dogs treated at 99 (Group $A \rightarrow E$ ) and 211 (Groups $\mathrm{E}, \mathrm{E} \rightarrow \mathrm{A}$, and $\mathrm{A}+\mathrm{E}$ ) days post-E. canis infection were $E$. canis PCR negative within 7 days of initiating doxycycline therapy and remained PCR negative for the duration of the study, including the post-immunosuppression period (Table 2). Despite immunosuppression, neither A. platys nor E. canis DNA was PCR amplified from blood, lymph node or bone marrow of any doxycycline-treated dog with complete agreement between the two laboratories performing the PCR testing.

In contrast, those dogs in Groups $\mathrm{E}, \mathrm{A} \rightarrow \mathrm{E}, \mathrm{E} \rightarrow \mathrm{A}$ and $\mathrm{E}$ + A that were not treated with doxycycline remained $E$. canis PCR positive both pre- and post-immunosuppression in blood and lymph node, however fewer $E$. canis PCR positive results were obtained with bone marrow (Table 2). The results of both the conventional and realtime PCR assays were in complete agreement for the preimmunosuppression samples, but demonstrated only $82 \%$ agreement on the post-immunosuppression samples. Only one untreated, $A$. platys infected dog from Group $\mathrm{A}+\mathrm{E}$

Table 2 Compiled real-time and conventional E. canis PCR results pre- and post-immunosuppression.

\begin{tabular}{llclcc}
\hline & \multicolumn{2}{c}{$\begin{array}{c}\text { Pre-immunosuppression } \\
\text { (Day 400) }\end{array}$} & & $\begin{array}{c}\text { Post-immunosuppression } \\
\text { (Day 414 and 420) }\end{array}$ \\
\hline $\begin{array}{l}\text { Group } \\
(\mathbf{n}=\mathbf{3})\end{array}$ & Blood & Lymph Node & Blood & Lymph Node & Bone Marrow \\
\hline Group E & $3 / 3$ & $3 / 3$ & $3 / 3$ & $2 / 2$ & $2 / 3$ \\
\hline Group E+doxy & $0 / 3$ & $0 / 3$ & $0 / 3$ & $0 / 2$ & $0 / 3$ \\
\hline Group A $\rightarrow$ E & $3 / 3$ & $3 / 3$ & $3 / 3^{*}$ & $2 / 2$ & $1 / 3$ \\
\hline Group A $\rightarrow$ E+doxy & $0 / 3$ & $0 / 3$ & $0 / 3$ & $0 / 3$ & $0 / 3$ \\
\hline Group E $\rightarrow$ A & $3 / 3$ & $3 / 3$ & $3 / 3$ & $3 / 3$ & $3 / 3^{*}$ \\
\hline Group E $\rightarrow$ A+doxy & $0 / 3$ & $0 / 3$ & $0 / 3$ & $0 / 3$ & $0 / 3$ \\
\hline Group A+E & $3 / 3$ & $3 / 3$ & $3 / 3^{*}$ & $3 / 3$ & $2 / 3^{*}$ \\
\hline Group A+E+doxy & $0 / 3$ & $0 / 3$ & $0 / 3$ & $0 / 3$ & $0 / 2$ \\
\hline
\end{tabular}

Untreated control dogs are compared to the doxycycline treated dogs (+doxy). Doxycycline was administered between days 211 and 238 . Dexamethasone was administered between days 410 and 414 . (Number of dogs testing PCR positive/number tested in each group.*Indicates those groups where PCR results for individual samples disagreed between the methods.) 
tested PCR positive for $A$. platys DNA in blood pre-immunosuppression, and was PCR positive in blood, lymph node and bone marrow post-immunosuppression.

\section{Discussion}

Results of this study demonstrate that concurrent or sequential infection with $A$. platys and $E$. canis can impact the hematological changes induced by these pathogens and can also alter the anticipated host immune response that would be induced following exposure to only one organism. Simultaneous infection with E. canis and $A$. platys in dogs resulted in a more pronounced anemia and thrombocytopenia, when compared to the sole infection with either pathogen. Both sequential and simultaneous infections with $A$. platys and $E$. canis produced an enhanced immune response to $A$. platys when compared to infection with $A$. platys alone. Also, co-infection with $E$. canis and $A$. platys appeared to result in a more persistent $A$. platys infection than was observed in those dogs that were infected only with $A$. platys. While the dogs in this study were infected experimentally, there is substantial evidence to support natural exposure to and infection with multiple tick-borne pathogens in dogs [5-8,10,11,23-27]. Under natural conditions, tick transmission potentially influences the course of infection and clinical manifestations, and is therefore a limitation of experimental infection studies. It is likely that co-infection or sequential infections contribute to some of the "atypical" manifestations that have been historically and clinically attributed to single pathogen infections.

In this study, the hematologic effects of infection with only $A$. platys or only $E$. canis were similar to those previously reported [28-31]. The cyclic nature of the thrombocytopenia reported in A. platys infected dogs was not clearly demonstrated in this study due to the comparatively low frequency (e.g. twice weekly) in which platelet concentration was measured, and due to the effect of averaging platelet concentrations from multiple dogs per study group at a point in time. When compared directly, the initial decrease in platelet concentrations ( day $10 \mathrm{PI}$ ) occurred more rapidly in dogs infected with only $A$. platys, as compared to dogs infected with only $E$. canis. This suggests that each of these organisms may induce pathophysiologically different mechanisms that contributed to the thrombocytopenia documented in these dogs. However, the more rapid onset of thrombocytopenia in A. platys infected dogs may reflect a difference in either the strain, dosage or the specific isolate of the organisms used in this study. Nevertheless, compared to E. canis, which induces thrombocytopenia in association with the development of anti-platelet antibodies, $A$. platys directly infects platelets and may have a more immediate effect on the platelet circulating half-life [32-34].
An unexpected alteration in the pattern of seroconversion occurred in dogs that were initially infected with $A$. platys and later challenged with $E$. canis. Following $E$. canis challenge infection, there was a dramatic increase in anti-Anaplasma antibodies; even for one dog in which $A$. platys serum antibodies were no longer detectable at the time of $E$. canis infection. In addition, there was no molecular evidence (PCR positivity) that $A$. platys organisms were present in the circulation of these dogs at the time this increase in Anaplasma serum antibodies occurred. The sensitivity and specificity of the ELISA for antibodies to Anaplasma and Ehrlichia species has also been shown to be high, reducing the likelihood of cross-reacting or false positive results [35]. This finding suggests that infection with $E$. canis, and potentially other pathogenic organisms, can induce an immunogenic effect that results in an increased anamnestic response to previously recognized antigens, in addition to a specific humoral immune response to $E$. canis. This result is potentially consistent with previous findings which demonstrated that acute $E$. canis infections do not result in immunosuppression in the dog [36].

This study is the first to report the long-term serologic and PCR results for dogs experimentally infected with $A$. platys. Previous $A$. platys experimental studies reported on the acute phase of infection; with dogs being monitored for a maximum of 75 days PI independent of treatment $[29,30]$. Immunological clearance of A. platys was supported in this study by the progression from PCR positive to PCR negative blood analyses by day 160 PI in all dogs infected only with A. platys. All dogs appeared to have cleared their infection prior to antibiotic treatment. These findings support prior clinical impressions that most A. platys strains in the United States are considered to cause minimal clinical disease, despite concurrent documentation of thrombocytopenia [37]. However, isolates from other parts of the world are reported to induce a more severe disease in dogs $[5,30]$. This study was limited to the strains of $A$. platys and $E$. canis available for establishing the experimental infection and the results of single, sequential and simultaneous infections may differ depending upon the strain encountered in nature. Likewise, all inoculums were prepared stored and administered in an identical manner however, undetermined variability in the infectious dose administered to each dog could have influenced the results. As dogs co-infected with A. platys and E. canis in this study developed a more persistent infection in conjunction with more severe thrombocytopenia and anemia, clinicians investigating natural infection due to A. platys should consider the potential influence of other known or unknown tick-borne pathogens.

In this study, similar to several previous studies utilizing experimental infections, doxycycline was found to be 
an efficacious therapy for E. canis infection when administered for four weeks $[17,38]$. Those dogs treated at 99 (Group $\mathrm{A} \rightarrow \mathrm{E}$ ) and 211 (Groups $\mathrm{E}, \mathrm{E} \rightarrow \mathrm{A}$, and $\mathrm{A}+\mathrm{E}$ ) days post-E. canis infection were $E$. canis PCR negative within 7 days of beginning doxycycline therapy. For those $E$. canis infected dogs that did not receive doxycycline, E. canis DNA could be found through the last time point tested by PCR (day 420 of the study) with blood and lymph node samples being more reliable sources for testing than bone marrow, similar to a previous studies of naturally infected dogs [7,27].

Dexamethasone-induced immunosuppression resulted in a marked increase in the platelet counts for all dogs, which was more pronounced in chronically thrombocytopenic dogs infected with E. canis. Multiple mechanisms have been proposed for the thrombocytopenia associated with $E$. canis infections including increased platelet consumption, splenic sequestration and immune-mediated mechanisms associated with increased platelet destruction $[1,33,39]$. If immunosuppression was able to inhibit the immune-mediated destruction and the removal of platelets by tissue macrophages, the rapid rise and fall in platelet counts before and after corticosteroid administration may reflect an ongoing hyperplastic bone marrow response, which could potentially lead to hypoplastic bone marrow (exhaustion) in the chronic phase of canine ehrlichiosis $[40,41]$. The use of immunosuppressive corticosteroids for treatment of in immune-mediated thrombocytopenia must be considered carefully when a dog is knowingly or unknowingly infected with a pathogen. Severe immunosuppression in dogs with chronic, undiagnosed infections could contribute to highly variable clinical outcomes, including death.

\section{Conclusions}

This study was designed to evaluate the influence of simultaneous or sequential infections with $A$. platys and E. canis in dogs as compared to dogs inoculated with either pathogen alone. The study identified differences in the hematological and serological parameters and in the duration of infection during simultaneous co-infection or after inducing a sequential infection. Under natural conditions, it is not always clinically possible to know the variety of organisms dogs are exposed to or infected with, particularly in those regions of the world where a spectrum of vectors and multiple pathogens are endemic. Awareness and prevention of tick-borne and other vector-borne infections, using acaracides and other preventive modalities are clearly important. Diagnostically, co-infection should be considered in those dogs with atypically severe or unusual clinical presentations.

\section{Acknowledgements}

The authors would like to acknowledge Kristen DeBisceglie, Brendon Thatcher, Phyllis Tyrrell, and Jancy Hanscom for assistance with serologic and molecular testing at IDEXX, and Del Philips, LeeAnn Eddleman, Loree Haines, Claire Webster, and Elizabeth Chatelain for their veterinary technical assistance at LSU. This study was funded by IDEXX Laboratories, Inc. Publication of this thematic series has been sponsored by Bayer Animal Health $\mathrm{GmbH}$.

\section{Author details}

${ }^{1}$ Louisiana State University, School of Veterinary Medicine, Baton Rouge, LA USA. ${ }^{2}$ IDEXX Laboratories, Inc. Westbrook, ME, USA. ${ }^{3}$ Western University, College of Veterinary Medicine, Pomona, CA, USA. ${ }^{4}$ North Carolina State University, College of Veterinary Medicine, Raleigh, NC, USA.

\section{Authors' contributions}

The authors from IDEXX have been working with Drs. Breitschwerdt, Diniz and Gaunt for a number of years on vector-borne diseases and collaborated to design, analyze and interpret the data generated in this study. MJB and SDG drafted and revised the manuscript. All authors critically reviewed and approved the final manuscript.

\section{Competing interests}

MJB, BAS, LL and RC are employees of IDEXX Laboratories, Inc. EBB is a consultant for IDEXX Laboratories, Inc. PPVD was funded as a research postdoctoral fellow in the Intracellular Pathogens Research Laboratory at North Carolina State University, which EBB directs. SG has received funding from IDEXX Laboratories, Inc. within the last 5 years.

Received: 4 February 2010 Accepted: 8 April 2010

Published: 8 April 2010

\section{References}

1. Greene CE: Infectious Diseases of the Dog and Cat New York: Elsevier Health Sciences, 32006

2. Stich RW, Schaefer JJ, Bremer WG, Needham GR, Jittapalapong S: Host surveys, ixodid tick biology and transmission scenarios as related to the tick-borne pathogen, Ehrlichia canis. Vet Parasitol 2008, 158:256-273.

3. Bowman D, Little SE, Lorentzen L, Shields J, Sullivan MP, Carlin EP: Prevalence and geographic distribution of Dirofilaria immitis, Borrelia burgdorferi, Ehrlichia canis, and Anaplasma phagocytophilum in dogs in the United States: results of a national clinic-based serologic survey. Vet Parasitol 2009, 160:138-148.

4. Simpson RM, Gaunt SD, Hair JA, Kocan KM, Henk WG, Casey HW: Evaluation of Rhipicephalus sanguineus as a potential biologic vector of Ehrlichia platys. Am J Vet Res 1991, 52:1537-1541.

5. Harrus S, Aroch I, Lavy E, Bark H: Clinical manifestations of infectious canine cyclic thrombocytopenia. Vet Rec 1997, 141:247-250.

6. Cardoso L, Tuna J, Vieira L, Yisaschar-Mekuzas Y, Baneth G: Molecular detection of Anaplasma platys and Ehrlichia canis in dogs from the North of Portugal. Vet J 2008, 183:232-3.

7. Gal A, Loeb E, Yisaschar-Mekuzas Y, Baneth G: Detection of Ehrlichia canis by PCR in different tissues obtained during necropsy from dogs surveyed for naturally occurring canine monocytic ehrlichiosis. Vet $\lrcorner$ 2008, 175:212-217

8. Diniz PPVP, Beall MJ, Omark K, Chandrashekar R, Daniluk DA, Cyr KE, Koterski JF, Robbins RG, Lalo PG, Hegarty BC, Breitschwerdt EB: High prevalence of tick-borne pathogens in dogs from an Indian reservation in Northeastern Arizona. Vector Borne Zoonotic Dis .

9. Yabsley MJ, McKibben J, Macpherson CN, Cattan PF, Cherry NA, Hegarty BC, Breitschwerdt EB, O'Connor T, Chandrashekar R, Paterson T, Perea ML, Ball G, Friesen S, Goedde J, Henderson B, Sylvester W: Prevalence of Ehrlichia canis, Anaplasma platys, Babesia canis vogeli, Hepatozoon canis, Bartonella vinsonii berkhoffii, and Rickettsia spp. in dogs from Grenada. Vet Parasitol 2008, 151:279-285

10. Kordick SK, Breitschwerdt EB, Hegarty BC, Southwick KL, Colitz CM, Hancock SI, Bradley JM, Rumbough R, McPherson JT, MacCormack JN: Coinfection with multiple tick-borne pathogens in a Walker Hound kennel in North Carolina. J Clin Microbiol 1999, 37.2631-2638. 
11. Beall MJ, Chandrashekar R, Eberts MD, Cyr KE, Diniz PP, Mainville C, Hegarty BC, Crawford JM, Breitschwerdt EB: Serological and molecular prevalence of Borrelia burgdorferi, Anaplasma phagocytophilum, and Ehrlichia species in dogs from Minnesota. Vector Borne Zoonotic Dis 2008, 8:455-464.

12. Glaze MB, Gaunt SD: Uveitis associated with Ehrlichia platys infection in a dog. J Am Vet Med Assoc 1986, 189:916-917.

13. Baker DC, Simpson M, Gaunt SD, Corstvet RE: Acute Ehrlichia platys infection in the dog. Vet Pathol 1987, 24:449-453.

14. Eddlestone SM, Gaunt SD, Neer TM, Boudreaux CM, Gill A, Haschke E, Corstvet RE: PCR detection of Anaplasma platys in blood and tissue of dogs during acute phase of experimental infection. Exp Parasitol 2007, 115:205-210.

15. Eddlestone SM, Neer TM, Gaunt SD, Corstvet R, Gill A, Hosgood G, Hegarty B, Breitschwerdt EB: Failure of imidocarb dipropionate to clear experimentally induced Ehrlichia canis infection in dogs. J Vet Intern Med 2006, 20:840-844.

16. Gaunt SD, Corstvet RE, Berry CM, Brennan B: Isolation of Ehrlichia canis from dogs following subcutaneous inoculation. J Clin Microbiol 1996, 34:1429-1432.

17. Eddlestone S, Diniz PPVP, Neer TM, Gaunt SD, Corstvet R, Cho D, Hosgood G, Hegarty B, Breitschwerdt EB: Doxycycline clearance of experimentally induced chronic Ehrlichia canis infection in dogs. J Vet Intern Med 2007, 21:1237-42.

18. Stillman B, DeBisceglie K, Bradley J, Saucier J, Breitschwerdt EB, Gaunt SD, O'Connor T, Chandrashekar R: Experimental E. canis infection in dogs: Antibody response pre- and post-treatment [abstract]. JVIM 2008, 22:781-782.

19. IDEXX Laboratories, Inc. Detection of Anaplasma platys. US Patent 2009, 7:507-789.

20. Bell CA, Patel $R$ : A real-time combined polymerase chain reaction assay for the rapid detection and differentiation of Anaplasma phagocytophilum, Ehrlichia chaffeensis, and Ehrlichia ewingii. Diagn Microbiol Infect Dis 2005, 53:301-306.

21. Birkenheuer AJ, Levy MG, Breitschwerdt EB: Development and evaluation of a seminested PCR for detection and differentiation of Babesia gibsoni (Asian genotype) and B. canis DNA in canine blood samples. J Clin Microbiol 2003, 41:4172-4177.

22. Dawson JE, Biggie KL, Warner CK, Cookson K, Jenkins S, Levine JF, Olson JG: Polymerase chain reaction evidence of Ehrlichia chaffeensis, an etiologic agent of human ehrlichiosis, in dogs from southeast Virginia. Am J Vet Res 1996, 57:1175-1179.

23. Breitschwerdt EB, Hegarty BC, Hancock SI: Sequential evaluation of dogs naturally infected with Ehrlichia canis, Ehrlichia chaffeensis, Ehrlichia equi, Ehrlichia ewingii, or Bartonella vinsonii. J Clin Microbiol 1998, 36:2645-2651.

24. de Paiva Diniz PP, Schwartz DS, de Morais HS, Breitschwerdt EB: Surveillance for zoonotic vector-borne infections using sick dogs from southeastern Brazil. Vector Borne Zoonotic Dis 2007, 7:689-697.

25. Gal A, Harrus S, Arcoh I, Lavy E, Aizenberg I, Mekuzas-Yisaschar Y, Baneth G: Coinfection with multiple tick-borne and intestinal parasites in a 6 week-old dog. Can Vet J 2007, 48:619-622.

26. Mylonakis ME, Koutinas AF, Baneth G, Polizopoulou Z, Fytianou A: Mixed Ehrlichia canis, Hepatozoon canis, and presumptive Anaplasma phagocytophilum infection in a dog. Vet Clin Pathol 2004, 33:249-251.

27. Mylonakis ME, Koutinas AF, Breitschwerdt EB, Hegarty BC, Billinis CD, Leontides LS, Kontos VS: Chronic canine ehrlichiosis (Ehrlichia canis): a retrospective study of 19 natural cases. J Am Anim Hosp Assoc 2004, 40:174-184.

28. de Castro MB, Machado RZ, de Aquino LP, Alessi AC, Costa MT: Experimental acute canine monocytic ehrlichiosis: clinicopathological and immunopathological findings. Vet Parasitol 2004, 119:73-86.

29. Harvey JW, Simpson CF, Gaskin JM: Cyclic thrombocytopenia induced by a Rickettsia -like agent in dogs. J Infect Dis 1978, 137:182-188.

30. Kontos VI, Papadopoulos O, French TW: Natural and experimental canine infections with a Greek strain of Ehrlichia platys. Vet Clin Pathol 1991, 20:101-105.

31. Reardon MJ, Pierce KR: Acute experimental canine ehrlichiosis. I. Sequential reaction of the hemic and lymphoreticular systems. Vet Pathol 1981, 18:48-61.

32. French TW, Harvey JW: Canine infectious cyclic thrombocytopenia (Ehrlichia platys infection in dogs) New York: Pergamon Press 1993.
33. Harrus S, Waner T, Weiss DJ, Keysary A, Bark H: Kinetics of serum antiplatelet antibodies in experimental acute canine ehrlichiosis. Vet Immunol Immunopathol 1996, 51:13-20.

34. Waner T, Harrus S, Weiss DJ, Bark H, Keysary A: Demonstration of serum antiplatelet antibodies in experimental acute canine ehrlichiosis. Vet Immunol Immunopathol 1995, 48:177-182.

35. Chandrashekar RMC, Beall MJ, O'Connor T, Eberts M, Alleman AR, Gaunt SD, Breitschwerdt EB: Performance of the in-clinic SNAP ${ }^{\circledR} 4 D x^{\circledast}$ test for detection of antibodies to Anaplasma phagocytophilum, Ehrlichia canis, Borrelia burgdorferi and Dirofilaria immitis antigen in dogs. AJVR .

36. Hess PR, English RV, Hegarty BC, Brown GD, Breitschwerdt EB: Experimental Ehrlichia canis infection in the dog does not cause immunosuppression. Vet Immunol Immunopathol 2006, 109:117-125.

37. Bradfield JF, Vore SJ, Pryor WH Jr: Ehrlichia platys infection in dogs. Lab Anim Sci 1996, 46:565-568.

38. Schaefer JJ, Kahn J, Needham GR, Rikihisa Y, Ewing SA, Stich RW: Antibiotic clearance of Ehrlichia canis from dogs infected by intravenous inoculation of carrier blood. Ann N Y Acad Sci 2008, 1149:263-269.

39. Waner T, Leykin I, Shinitsky M, Sharabani E, Buch H, Keysary A, Bark H, Harrus S: Detection of platelet-bound antibodies in beagle dogs after artificial infection with Ehrlichia canis. Vet Immunol Immunopathol 2000, 77:145-150.

40. Harrus S, Waner T, Bark H, Jongejan F, Cornelissen AW: Recent advances in determining the pathogenesis of canine monocytic ehrlichiosis. J Clin Microbiol 1999, 37:2745-2749.

41. Reardon MJ, Pierce KR: Acute experimental canine ehrlichiosis. II. Sequential reaction of the hemic and lymphoreticular system of selectively immunosuppressed dogs. Vet Pathol 1981, 18:384-395.

doi:10.1186/1756-3305-3-33

Cite this article as: Gaunt et al:: Experimental infection and co-infection of dogs with Anaplasma platys and Ehrlichia canis: hematologic, serologic and molecular findings. Parasites \& Vectors 2010 3:33.

\section{Submit your next manuscript to BioMed Central and take full advantage of:}

- Convenient online submission

- Thorough peer review

- No space constraints or color figure charges

- Immediate publication on acceptance

- Inclusion in PubMed, CAS, Scopus and Google Scholar

- Research which is freely available for redistribution
C Biomed Central 